\title{
Immunological Aspects Related to the Pathophysiology of Multiple Sclerosis and the Possible Checkpoints for Target Therapy: An Integrative Literature Review
}

Ana Clara Loyola de Aguiar Andrade ${ }^{1}$, Diego Rodrigues Naves Barbosa Lacerda ${ }^{1}$, Gabriela Teixeira Argondizzi ${ }^{1}$, Mateus Borges Soares ${ }^{1}$, Pedro Teixeira Meireles ${ }^{1}$, Samilla Chaves Siqueira ${ }^{1}$, Ana Karina Marques Salge ${ }^{3}$, George Kemil Abdalla ${ }^{2}$ and Douglas Reis Abdalla ${ }^{1,2 *}$

${ }^{1}$ University of Uberaba, Brazil

${ }^{2}$ Faculty of Human Talents, Brazil

${ }^{3}$ Nursing School of the Federal University of Goiás - FEN/UFG, Brazil

*Corresponding author: Douglas Reis Abdalla, Medicine Student, University of Uberaba, Health Courses, Faculty of Human Talents, Uberaba, Minas Gerais, Brazil.
Received Date: April 08,2021

Published Date: April 22, 2021

\begin{abstract}
Multiple sclerosis (MS) is the most common chronic demyelinating disease of the central nervous system (CNS), with an autoimmune inflammatory mechanism, but the immunological analysis, described in the pathogenesis of the disease, is not attributed or significantly placed in the universally used criteria. In this regard, this literature review aims to highlight immunological aspects that can be attributed both to the diagnosis and treatment of MS. In this sense, this review compiled and interpreted 41 articles found published between 2010 and 2020 , indexed on the platforms: PubMed, Scielo and LILACS. Of these only 6 articles contained the proposed inclusion criteria. It was possible to show that CD4 T lymphocytes are activated by antigen presenting cells (microglia and B lymphocytes), adopting Th1 profiles (producing IFN- $\gamma$ and TNF- $\alpha$ ) and Th17 (producing IL-17 and IL-22); from the activation of B lymphocytes, they are differentiated into plasmocytes that will produce IgG4; due to the action of autoreactive lymphocytes, there is an inflammation at the neuronal level that triggers demyelination. In this sense, it is concluded that there is a need to encourage more research regarding the reduction of the inflammatory response in relation to MS and also the encouragement of clinical studies that are already being followed up, given the positive results presented by them and an improvement in the quality of life of their patients.
\end{abstract}

Keywords: Multiple Sclerosis; Brain Diseases; Encephalomyelitis; Immunology; Macrophages; Lymphocytes; Interleukins; Autoimmunity

\section{Introduction}

Multiple sclerosis (MS) is the most common chronic demyelinating disease of the central nervous system (CNS), with an autoimmune inflammatory mechanism, affecting in the form of characteristic lesions called "plaques", the white substance of the brain stem, cerebellum, spinal cord and optic nerve [1,2]. The tissue damage in MS results from a complex and dynamic interaction between the immune system, glial cells and neurons. However, this autoimmune inflammatory aggression, characterized by a lymphocytic infiltrate that crosses the blood-brain barrier, over the myelin sheath of the CNS axons, leads to the loss of trophic factors produced by this protective layer, promoting a permanent axonal degeneration, resulting in a series of motor and sensory signs and 
symptoms $[3,4]$. Worldwide, it affects about 2.5 million people and within that amount, 35 thousand are Brazilian [5]. Among those affected, most of them are young adults between 20 and 40 years of age. In Europe and North America, MS is considered a highly prevalent disease, since for every 100,000 inhabitants, 60 to 200 sick individuals are found [6]. This reality does not correspond to South America, which has a rate of less than 5 cases per 100,000 inhabitants, that is a low prevalence [7].

In Brazil, the incidence is about 5-30 cases per 100,000 inhabitants, but varies according to the region [6]. In addition, there are studies that show that the disease predominates in white women with the outbreak-remissive form in the national and international context. However, in a study in Rio de Janeiro, 31.8\% of black patients were found. In general, MS is a rare disease among blacks, it is believed that the largest number of blacks in Brazilian studies is due to a change in genetic susceptibility as less favored socioeconomic conditions and to the racial mixture present in the Brazilian people [8].

Regarding the etiology of MS, this remains unknown, however, it is believed to be caused by immune dysregulation triggered by genetic and environmental factors [9]. Although MS is not an inherited disease, there is a strong genetic component in its etiology, as evidenced by the grouping of MS cases in families. The risk of MS among first-degree relatives with MS is 10 to 50 times higher than the general population (absolute risk 2 to 5\%); the agreement rate in monigotic twins is about one third $[10,11]$. Linkage analysis studies have revealed several genetic sites as risk factors, with the HLA DR15/DQ6 allele being the main histocompatibility complex (MHC) the strongest $[12,13]$. Alleles of the interleukin-2 receptor alpha gene (IL2RA) and the interleukin-7 receptor alpha gene (IL7RA) have also been identified as inheritable risk factors $[13,14]$ However, most of the genetic factors underlying susceptibility have yet to be defined. In addition, in the autoimmune hypothesis, the demyelization process begins with activation of peripheral CD4 $+\mathrm{T}$ cells with reactivity to myelin. However, in this hypothesis, unlike the viral infectious hypothesis, the cause of activation is unknown. Activated CD $4+\mathrm{T}$ cells cross the blood-brain barrier, genetically altered, towards the Central Nervous System, aided by an increase in the number of adhesion molecule receptors expressed by endothelial cells [15]. According to Dos Santos et al (2005) [16], myelin autoantigens, especially Basic Myelin Protein and Myelodendritic Glycoprotein, are presented by macrophages to these CD 4 + T cells, triggering a cascade that releases inflammatory mediators and culminates in the injury, or even destruction, the myelin sheath. In addition to the action of T cells, we observed the participation of B cells in the infiltrate and the action of antibodies on the surface of the myelin that acts in the demyelination process, in addition to molecules of the Complement System [17]. Currently, early treatment of MS is expected to be related to preventing the patient's long-term disability. These treatments can be immunosuppressive or immunomodulatory, which implies that it is necessary to control inflammation and, consequently, disease activity. The closest that has ever come to a potential cure has been immune reconstitution therapies [18]. A concept in treatment has recently been developed that suggests that clinical recurrences of the disease are a small part of the disease, which is "no evidence of disease activity" or NEDA. This fact arose from the perception that scholars had about continuous inflammatory activity. It was observed that even cerebral atrophy occurred even at times when the disease was not clearly active [18].

This literature review aims to highlight immunological aspects that can be attributed both to the diagnosis and treatment of MS.

\section{Methodology}

It is an integrative literature review, which allows the evaluation and synthesis of evidence and knowledge about the phenomenon studied, by understanding its current state, as well as proposing effective interventions in health care. For this, six methodological steps of the review were carried out. The first stage consisted in the elaboration of the hypothesis starting from a guiding question of the research, that is, identification of the problem, search engine to be adopted and the descriptors or keywords. The second step was the establishment of inclusion and exclusion criteria for the articles to be selected to compose the sample. The third stage was an exploratory reading of the abstracts, making a pre-selection of the studies. In the fourth stage, an analytical reading of the studies was carried out in order to gather, analyze and categorize the information pertinent to the investigated problem. In the fifth stage, the results were interpreted. In the sixth and final stage, the synthesis and presentation of the identified results was promoted, as well as recommendations for further studies.

Once the theme was defined, the following guiding question was elaborated: what is the evidence in the evolution of scientific production on approaches that can modify the immune response in patients with multiple sclerosis? The research on scientific production was carried out in 2020, through a bibliographic survey in the databases of the National Institutes of Health PubMed, Electronic Scientific Online Library - Scielo and Latin American and Caribbean Health Sciences Information System - LILACS , in order to map articles relevant to the theme. For this purpose, the keywords multiple sclerosis, encephalopathies, encephalomyelitis, immunology, macrophages, lymphocytes, interleukins, autoimmunity, isolated and in association, were used in order to find similar themes published until today. Altogether, with the association of keywords, 41 articles were found. These were filtered with respect to Portuguese, English and Spanish, publications from January 2010 to June 2020, full texts online, being case reports, case series, original articles, retrospective, prospective and observational studies, with themes related to mechanisms related to multiple sclerosis immunopathogenesis and the main check points as immunotherapeutic targets (figure 01). Literature review articles, publications before January 2010 and 
after December 2020, languages other than English, Portuguese or Spanish, and articles do not complete in full or with themes that did not resemble the proposal in the work were also excluded (Figure 1).

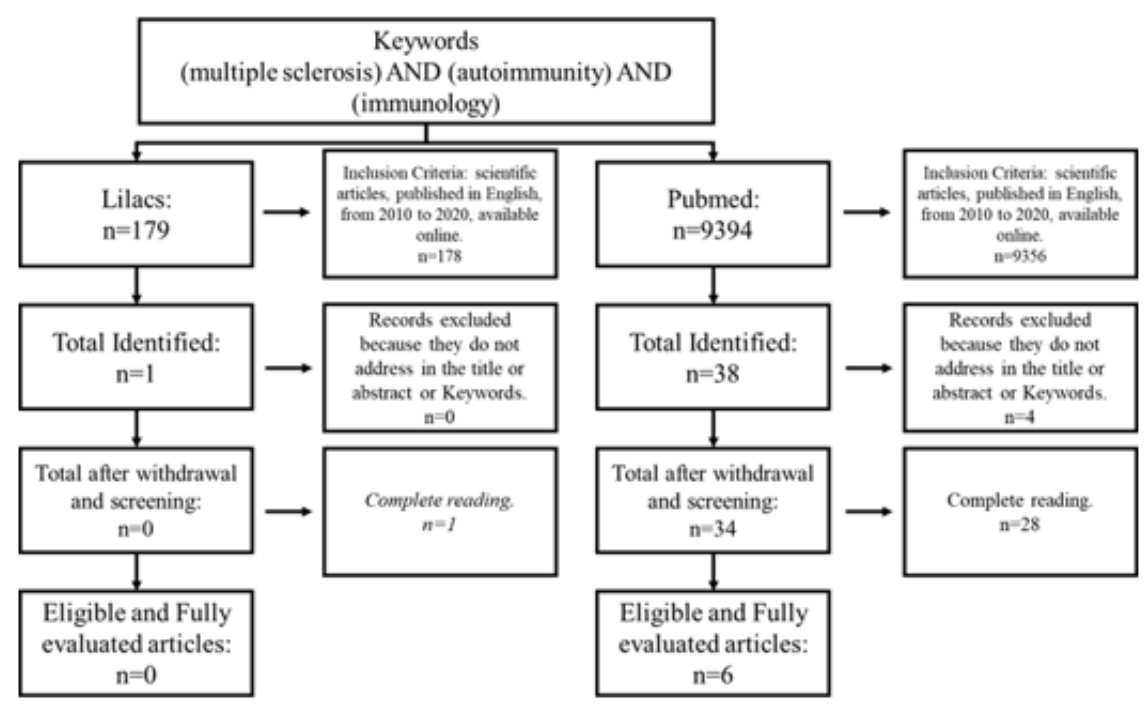

Figure 1: Flowchart of the selection process of articles found and included in the review, in Lilacs and PubMed / Medline - Uberaba / MG, 2020

\section{Results and Discussion}

In the time frame established for this study (2010-2020), 6 publications were found and analyzed. In 2014, 2015 and 20171 article (50\%) was published each year respectively, in 2018 there are 2 publications $(33.3 \%)$ and in 2019 only 1 publication in the year $(16.7 \%)$. The publications resulted from different journals: Acta Medica Iranica, MAbs, Multiple Sclerosis Journal, Frontiers in immunology, JCI insight and Proceedings of the National Academy of Sciences (PNAS). Analyzing the study places of the articles, each one was carried out in a different location, being: Iran (16.6\%), Switzerland (16.6\%), USA (16.6\%), Canada (16.6\%), Australia (16.6\%) and Spain (16.6\%). Multiple Sclerosis (MS) is an autoimmune and neurodegenerative disease of the Central Nervous System (CNS) The pathophysiological process basically consists of an intense inflammatory process that evolves with demyelination and axonal loss [19]. This autoimmunity that evolves with inflammation is due to the imbalance of different subsets of T cells, especially those of the Th1 and Th17 profiles [20], associated with antibodies (IgG4) against myelin peptides [21].

Thus, currently, different aspects that explain this pathophysiology from infectious processes, autoimmune processes and genetic mutations. Based on these understandings, therapeutic measures have been developed, even if experimental, that can be used effectively for the clinical improvement of patients. An example of this is the clinical trial carried out with 13 patients, in which 10 received a complete therapeutic plan with specific T cells for the Epstein Barr virus (EBV), 4 with T cells with weak reactivity to the virus and 6 with high reactivity to the virus. Among those who received the dose of high reactivity, all had objective symptomatic neurological improvement, associated with reduction and fatigue. As for those who received low reactivity, only 25\% showed improvement similar to the other group [22]. This is explained because in other studies it has been shown that EBV-specific T cell therapy should kill EBV-infected B cells in the CNS and thus prevent disease progression and lead to clinical improvement. And the relationship with MS is that EBV infection may be the precursor to the inflammatory process in the CNS [22]. In this perspective of devising a method of symptom improvement and even reaching the cure of MS, a Canadian clinical study, in phase 2, with 24 individuals, evaluated the autologous transplantation of hematopoietic stem cells to treat the aggressive form of MS. The researchers found that there was an increase in Natural Killers (NK) cells providing initial co-stimulation that supports the induction of a Th17 response, but followed by cytotoxicity which limits these cells. Thus, these data suggest that the rapid reconstitution of NK cells after transplantation contributes to suppressing the resurgence of Th17 cells and thus reducing the inflammatory process, which is at the heart of MS [20]. In this sense of suppression of T cells for remission and treatment of MS, a North American study, randomized double blind clinical study, with 123 individuals, evaluated the efficacy and safety of the co-stimulatory block made by the CTLA4-Ig fusion protein abatacept, when associated with T lymphocyte in MS. In general, there was neither clinical improvement nor reduction in the number of classic lesions on magnetic resonance imaging of the skull. However, since there was no worsening of the patients' condition or the appearance of new lesions, these antibodies are potentially effective and there is a need for further studies [23]. 
As regarding to the use of monoclonal antibodies in the treatment and clinical improvement of patients with MS, a clinical study in phase 1, first developed on animals and already tested on 2 volunteers, showed that GNbAC1, a humanized IgG4 monoclonal antibody, targets the retrovirus envelope protein associated with MS, is promising and both animals and humans beyond its safety [24]. This specific study demonstrates the need for investment in research with monoclonal antibodies, mainly for safety and efficiency. Finally, it is worth mentioning that there are measures beyond direct therapy with the introduction of antibodies or transplants to improve the patient with MS. Randomized clinical trial, Iranian, carried out with 101 participants, of middle age, of both sexes, evaluated the effect of vitamin supplementation with the levels of cytokines and the genetic expression of patients already diagnosed with MS. It was concluded that $100 \%$ of the participants had a response to treatment, this being partial or total. Thus, evidencing that retinol and its metabolites have a promising possibility of preventing inflammatory processes in the CNS, in addition to the neurodegenerative process. This, therefore, shows regression of the disease [19].

In this sense, figure 02 summarizes the pathophysiological process of multiple sclerosis in terms of immunological aspects and possible checkpoints for the target therapy. The numbers 1 to 4 represent the etiopathogenesis of multiple sclerosis (MS). The letters $\mathrm{A}$ to $\mathrm{C}$ are the factors that modulate the immune response of MS. 1) In the presence of viral infections by Epstein-Barr (EBV) and multiple sclerosis-associated retrovirus (MSRV), an immune response is initiated. 2) LTCD4 are activated by antigen presenting cells (APC), such as microglia and B lymphocytes. They adopt a Th1 profile, producing IFN and TNF, and a Th17 profile, producing IL-17 and IL-22. 3) LB differentiate into plasmocytes, which will produce IgG4. 4) Neuronal inflammation occurs, which triggers demyelination due to the action of autoreactive lymphocytes. A) The IgG4 monoclonal antibody, GNbAC1, acts on the MSRV envelope protein. B) Abatacept CTLA4-Ig fusion protein performing costimulatory block when associated with LT. C) Retinol acting against inflammatory and neurodegenerative processes (Figure 2).

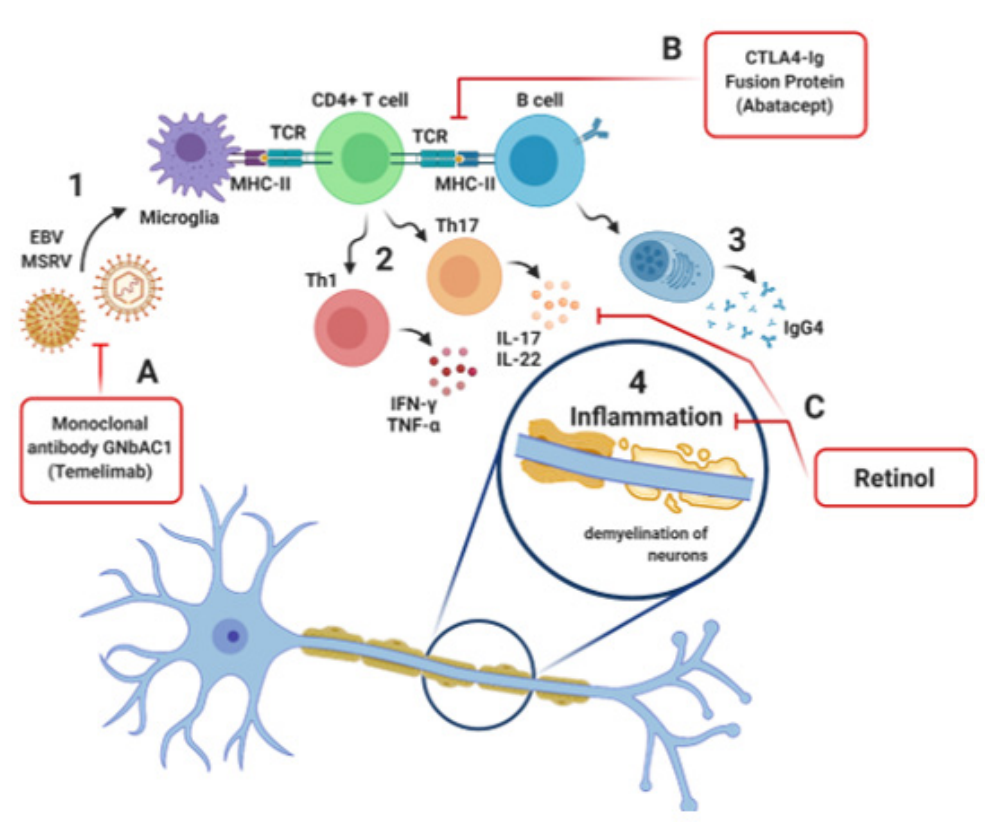

Figure 2: Representation of the pathophysiological process of multiple sclerosis: immunological aspects and possible checkpoints for the target therapy. Created with BioRender.com.

\section{Final Considerations}

MS is the most common chronic demyelinating disease of the central nervous system, resulting from a complex and dynamic interaction between the immune system, glial cells and neurons. However, it was evident that the criteria commonly used for the pathogenesis of MS lack immunological aspects that assist in the diagnosis and treatment of the disease. Therefore, it is concluded that there is a need for further research related to the immunological aspects attributed to the diagnosis and treatment of MS, which are linked to the reduction of the inflammatory response. Notwithstanding, clinical studies already underway lack incentive, given their promising results and the improvement in the quality of life of the patients involved.

\section{Acknowledgement}

None.

\section{Conflict of Interest}

No conflict of interest.

\section{References}

1. Neurological Disorders Collaborator Group (2017) Global, regional, and national burden of neurological disorders during 1990-2015: a 
systematic analysis for the Global Burden of Disease Study. Lancet Neurol 16(11): 877-897.

2. Brownlee WJ, Hardy TA, Fazekas F, Miller DH (2017) Diagnosis of multiple sclerosis: progress and challenges. Lancet 89: 1336-1346.

3. Lassmann H (2014) Mechanisms of white matter damage in multiple sclerosis. Glia 62: 1816-1830.

4. BFG, Bunyan RF (2011) Inflammatory Lucchinetti CF Popescu cortical demyelination in early multiple sclerosis. N Engl J Med 365: 2188-2197.

5. Associação Brasileira De Esclerose Múltipla (ABEM). Esclerose múltipla.

6. Larrussa, Giovanni Tognela (2015) The influence of the endocannabinoid system on the pathophysiology of multiple sclerosis. J Health Sci Inst.

7. Baggio, Bruna Finato (2011) Epidemiological profile of individuals with Multiple Sclerosis from a reference association. Neuroscience Magazine 19(3): 458-461.

8. Oliveira Enedina, M Annes, A S Oliveira, A A Gabbai (1999) [Multiple sclerosis. Clinical survey of 50 patients followed at the Ambulatory of Neurology UNIFESP-EPM]. Arq Neuropsiquiatr 57(1):51-55.

9. Ascherio, Alberto; Munger, Kassandra L (2007) Environmental risk factors for multiple sclerosis. Part II: Noninfectious factors. Ann Neurol 61(6): 504-513.

10. Kantarci OH (2008) Genetics and natural history of multiple sclerosis. Semin. Neurol 28(1): 7-16.

11. Barcellos, L F Oksenberg, J R Begovich, A B Martin, E R Schmidt, et al. (2003) HLA-DR2 dose effect on susceptibility to multiple sclerosis and influence on disease course. Am J Hum Genet 72(1): 710-716.

12. Sawcer S, Pirinen M, Spencer, C C A Patsopoulos, N A Moutsianas L et al. (2011) Genetic risk and a primary role for cell-mediated immune mechanisms in multiple sclerosis. Nature 476(7359): 214-219.

13. Hafler DA, Compston A, Sawcer S, Lander ES, Daly MJ et al. (2007) Risk alleles for multiple sclerosis identified by a genomewide study. $\mathrm{N}$ Engl J Med 357(9): 851-862.

14. Romagopalan SV, Maugeri NJ, Handunnetthi L et al. (2009) Expression of the Multiple Sclerosis- associated MHC class II allele HLADRB11501 is regulated by Vitamin D. PLoS Genetics 5(2):
15. Ransohoff, Richard M, Kivisäkk Pia, Kidd Grahame (2003) Three or more routes for leukocyte migration into the central nervous system. Nat Rev Immunol 3(7): 569-581.

16. Dos Santos A C, Barsante M M, Arantes R M E, Bernard C C A, Teixeira M M, et al. (2005) CCL2 and CCL5 mediate leukocyte adhesion in experimental autoimmune encephalomyelitis-an intravital microscopy study. J Neuroimmuno 162(1-2): 122-129.

17. Cabrera Angel, Collins, William C, SALGADO, Jesus F (2006) Determinants of individual engagement in knowledge sharing. The International Journal of Human Resource Management 17(2): 245-264.

18. Dobson Ruth, Giovannoni, Gavin (2019) Multiple sclerosis - a review European journal of neurology. Eur J Neurol 26(1): 27-40.

19. Harrirchian MH, Mohammadzadeh Honarvar N, Koohdani F, Bitarafan S, Siassi F et al. (2014) The effect of vitamin A supplementation on disease progression, cytokine levels and gene expression in multiple sclerotic patients study protocol for a randomized controlled trial. Acta Med Iran 52(2): 94-100.

20. Darlington PJ, Stopnicki B, Touil T, Doucet JS, Fawaz L et al. (2018) Natural Killer Cells Regulate Th17 Cells After Autologous Hematopoietic Stem Cell Transplantation for Relapsing Remitting Multiple Sclerosis. Front Immunol 7(9): 834.

21. Zubizarreta I, FlórezGrau G, Vila G, Cabezón R, España C, et al. (2019) PImmune tolerance in multiple sclerosis and neuromyelitis optica with peptide loaded tolerogenic dendritic cells in a phase $1 \mathrm{~b}$ trial. Proc Natl Acad Sci U S A 23116(17): 8463-8470.

22. Pender MP, Csurhes PA, Smith C, Douglas NL, Neller MA, et al. (2018) Epstein-Barr virus-specific $\mathrm{T}$ cell therapy for progressive multiple sclerosis. JCI Insight 153(22): 124-714.

23. Khoury SJ, Rochon J, Ding L, Byron M, Ryker K, et al. (2017) A randomized trial of abatacept (CTLA4-Ig) for relapsing-remitting multiple sclerosis. Mult Scler 23(5): 686-695.

24. Curtin F, Perron H, Kromminga A, Porchet H, Lang AB (2015) Preclinical and early clinical development of GNbAC1 a humanized IgG4 monoclonal antibody targeting endogenous retroviral MSRV-Env protein. MAbs $7(1): 265-275$. 